\title{
The low-mass diskless population of Corona Australis
}

\author{
B. López Martí1 ${ }^{1}$, L. Spezzi ${ }^{2}$, B. Merín ${ }^{3}$, M. Morales-Calderón ${ }^{1,4}$, H. Bouy ${ }^{3}$, D. Barrado y Navscués ${ }^{1}$, and J. Eislöffel ${ }^{5}$ \\ ${ }^{1}$ Laboratorio de Astrofísica Estelar y Exoplanetas, Dpto. Astrofísica, Centro de Astrobiología (LAEX-CAB), CSIC-INTA, \\ ESAC Campus, PO Box 78, 28261 Villanueva de la Cañada, Madrid, Spain \\ e-mail: belen@cab.inta-csic.es \\ 2 Research and Scientific Support Department, European Space Agency (ESTEC), PO Box 299, 2200 AG Noordwijk, \\ The Netherlands \\ 3 Herschel Science Centre, European Space Agency (ESAC), PO Box 78, 28691 Villanueva de la Cañada, Madrid, Spain \\ 4 Spitzer Science Center, California Institute of Technology, Pasadena, CA 91125, USA \\ 5 Thüringer Landessternwarte, Sternwarte 5, 07778 Tautenburg, Germany \\ Received 23 November 2009 / Accepted 12 March 2010
}

\section{ABSTRACT}

\begin{abstract}
We combine published optical and near-infrared photometry to identify new low-mass candidate members in an area of about 0.64 deg ${ }^{2}$ in Corona Australis with the $S$-parameter method. Five new candidate members of the region are selected. They have estimated ages between 3 and $15 \mathrm{Myr}$ and masses between 0.05 and $0.15 M_{\odot}$. With Spitzer photometry we confirm that these objects are not surrounded by optically thick disks. However, one of them is found to display excess at $24 \mu \mathrm{m}$, thus suggesting it harbors a disk with an inner hole. With an estimated mass of $0.07 M_{\odot}$ according to the SED fitting, this is one of the lowest-mass objects reported to possess a transitional disk. Including these new members, the fraction of disks is about $50 \%$ among the total Corona Australis population selected by the same criteria, lower than the $70 \%$ fraction reported earlier for this region. Even so, we find a ratio of transitional to primordial disks (45\%) very similar to the value derived by previous authors. This ratio is higher than for solar-type stars (5-10\%), suggesting that disk evolution is faster in the latter, and/or that the "transitional disk" stage is not such a short-lived step for very low-mass objects. However, this impression needs to be confirmed with better statistics.
\end{abstract}

Key words. stars: low-mass - stars: formation - stars: pre-main sequence - stars: luminosity function, mass function

\section{Introduction}

In the last two decades, our understanding of the formation and early evolution of low-mass stars and substellar objects has been remarkably improved, but there are still many unknowns regarding the exact mechanisms at play in these processes (see e.g. Whitworth et al. 2007, for a review). The census of the low-mass population for an increasing number of star-forming regions is important to address the problem of the universality of the initial mass function (IMF). The comparison of the disk fractions (that is, the number of sources with disks with respect to the total number of members) in clusters of similar and different ages also helps to constrain the timescales of disk evolution (e.g. Haisch et al. 2001). Moreover, the disk fraction and the spatial distribution of stars with and without disks provide important clues to understand the star-forming history and the disk evolution of a particular region.

Most of our current knowledge about these issues comes from the study of star-forming regions and young clusters at distances within 100-300 pc from the Sun (see the reviews collected in Reipurth 2008). However, in the vast majority of these studies the identification of cluster members is based on the detection of features related to accretion and circum(sub)stellar disks (e.g. $\mathrm{H} \alpha$ emission, infrared excess), or indicative of strong activity (e.g. hard X-ray emission). Thus, these surveys are likely to be missing a fraction of the young low-mass population, especially objects that have dissipated all or most of their disks, that are in quiescence at the moment of the observations, and/ or whose activity level lies below the detection threshold of current $\mathrm{X}$-ray surveys.
In a recent paper Comerón et al. (2009, hereafter CSL09) reported on a large-scale optical survey of the Lupus clouds, selected to include most of the areas surveyed in the Spitzer Legacy program "From molecular cores to planet-forming disks" $(c 2 d)$. Combining their data with 2MASS near-infrared photometry, these authors developed an analysis procedure based on a dimensionless parameter $S$ that allowed them to easily identify very low-mass members of the dark clouds as an excess in the expected S-parameter distribution of field cool dwarfs and giants (see Sect. 3, for details). In this way they were able to unveil a large population of objects belonging to Lupus I and III, which seems to be composed of very low-mass stars and brown dwarfs that have lost their inner disks on a timescale of a few Myr. The discovery of a substantial and even dominant population of thus far unnoticed members of one of our nearest star-forming regions stresses the important unknowns that still subsist in the observational characterization of young very low-mass objects. It also poses the important question of whether similar populations of yet unknown very low-mass members exist in other regions.

In this paper, we apply the $S$-parameter analysis to a set of optical and near-infrared observations of Corona Australis, another of the nearest regions with ongoing or recent intermediate and low-mass star formation. Our aim is the identification of new very low-mass candidate members of this dark cloud. We focus on the core in the direction of the intermediate-mass star R CrA, which contains a compact embedded stellar cluster known as the "R CrA cluster" or "the Coronet cluster" (Taylor \& Storey 1984). With an age of about $3 \mathrm{Myr}$, its distance has been estimated to be within 50-170 pc (see Neuhäuser \& Forbrich 2008 , for a review). The young stellar population in this region 
has been studied with different techniques, including $\mathrm{H} \alpha$ surveys (e.g. Marraco \& Ryndgren 1981), optical spectroscopy (e.g. Walter et al. 1997), near-infrared mapping (e.g. Wilking et al. 1992), mid-infrared surveys (e.g. Olofsson et al. 1999) and X-ray observations (e.g. Neuhäuser et al. 2000; Forbrich \& Preibisch 2007). Some brown dwarfs and brown dwarf candidates have been reported (Wilking et al. 1997; Fernández \& Comerón 2001; Bouy et al. 2004; López Martí et al. 2005). Very recently, this cloud has also been the target of Spitzer observations (SiciliaAguilar et al. 2008; López Martí et al., in preparation).

The structure of the paper is as follows: in Sect. 2 we present the data used in our analysis. Section 3 summarizes the basis of the $S$-parameter formalism, discusses the contamination expected in our sample and describes the object selection. Section 4 is a discussion of the membership status and the properties of our new candidate members. Finally, in Sect. 5 we draw our conclusions.

\section{Optical and near-infrared data}

The analysis presented here is based on the same set of optical observations of the Corona Australis star-forming region reported in López Martí et al. (2005). The survey was performed with the WFI mosaic camera at the MPG/ESO $2.2 \mathrm{~m}$ telescope of La Silla Observatory. It covered an area of about $0.64 \mathrm{deg}^{2}$, distributed in two fields of $34^{\prime} \times 34^{\prime}$, which are placed in the core of the Coronet cluster and on a region of lower cloud density to the South of it (Fig. 1). For the present work, only the broadband observations in the $R$ and $I$ filters were considered, which cover the dynamical ranges $11<R<22$ and $12<I<23$. The survey is complete down to $R \simeq 20 \mathrm{mag}$ and $I \simeq 19 \mathrm{mag}$. For details on the observations, data reduction and calibration, see López Martí et al. (2005) and references therein.

The optical data were cross-matched with $J H K s$ photometry from the 2MASS archive (Skrutskie at al. 2006), which is complete down to $J \simeq 16.5$. For the present analysis, we require that the objects are detected in all five RIJHKs bands. The total number of sources fulfilling this criterion amounts to 6136 . Our sample is complete down to a mass of about $0.015 M_{\odot}$ at a distance of $130 \mathrm{pc}$ and an age of $3 \mathrm{Myr}$, according to the Lyon models (Baraffe et al. 1998; Chabrier et al. 2000).

\section{Analysis}

\subsection{SED fitting}

The spectral energy distributions (SEDs) of the objects in our optical-NIR catalog were fitted to the model spectra of Hauschildt et al. (1999) and Allard et al. (2000) as explained in CSL09 (see also Spezzi et al. 2007). The observed magnitude at each band is expressed as

$m_{\mathrm{obs}}^{\lambda}=M_{\mathrm{synt}}^{\lambda}+A_{V} \cdot \frac{A_{\lambda}}{A_{V}}+S$

where $M_{\text {synt }}^{\lambda}$ is a synthetic magnitude in the same passband, computed from the model spectra, for a $1 R_{\odot}$ star at the same temperature; and $\mathrm{S}$ is a wavelength-independent scaling factor defined as

$S=5 \log D(p c)-5 \log R\left(R_{\odot}\right)-5$.

The ratio $A_{V} / A_{\lambda}$ is a constant given by the adopted extinction law (Cardelli et al. 1989, with $R_{V}=3.1$ ). A value of $\log g=4.0$,

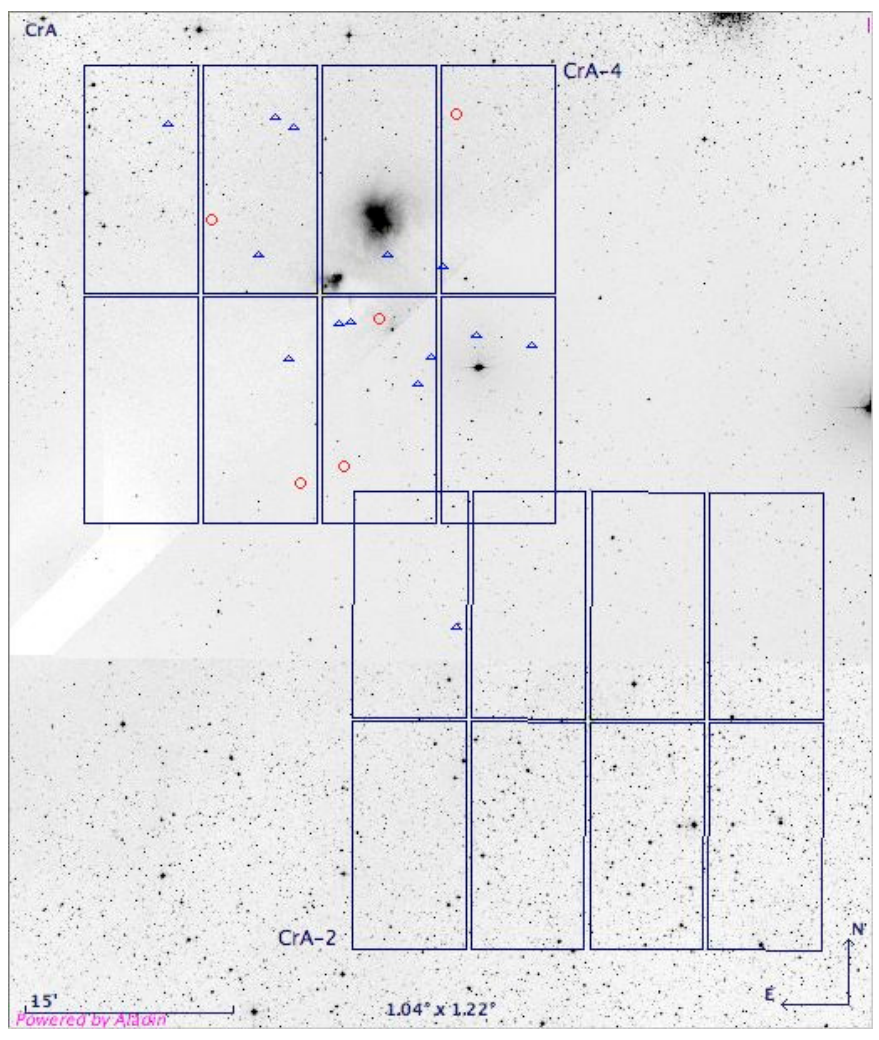

Fig. 1. Spatial location of the previously known candidate members (triangles) and the new candidates from this work (circles) within the Corona Australis dark cloud. Note that only those previous candidate members selected with the same criteria as our new candidates are plotted. The two WFI fields are also indicated. The objects are clustered around the intermediate-mass stars, which suggests that they belong to the Coronet cluster.

thought of being representative for young low-mass objects, was assumed for all the sources.

Assuming a certain $T_{\text {eff }}$ for each star, an equation of the form (1) was set for each passband and solved by least-squares to derive $A_{V}$ and $S$. The temperature that minimized the residual of the fit was then taken as the temperature of the object. The radius $R$ was computed from the corresponding value of $S$ for each source using Eq. (2) and a distance value of $130 \pm 20$ pc to the Corona Australis star-forming region (Marraco \& Ryndgren 1981; Neuhäuser \& Forbrich 2008). The luminosity $L$ was then computed with the relation $L=4 \pi \sigma R^{2} T_{\text {eff }}^{4}$, where $\sigma$ is the Stefan-Boltzmann constant. Finally, the best-fitting $T_{\text {eff }}$ and the derived luminosity were used to estimate stellar masses and ages from the evolutionary models and tracks by Baraffe et al. (1998) and Chabrier et al. (2000), again assuming a distance of $130 \pm 20 \mathrm{pc}$.

We note that the use of a different distance would modify the estimated radii, luminosities and ages, but not the derived effective temperatures, extinctions and $S$-parameter values. For instance, a distance of $170 \mathrm{pc}$, as suggested by Knude \& Høg (1998), would imply higher luminosities (by 0.23 in logarithmic scale), larger radii (by less than 0.5 for $R \leq 1.5 R_{\odot}$ ) and younger ages by about 2.5 Myr. However, because the ages derived using only photometry and isochrones, as in our case, intrinsically contain an uncertainty of a few Myr (e.g. Baraffe et al. 2002, 2009), the distance uncertainty is not a major issue for our parameter estimates.

The residuals for the best fit give in turn an estimate of the quality of the solution. We found that $94 \%$ of the objects have 


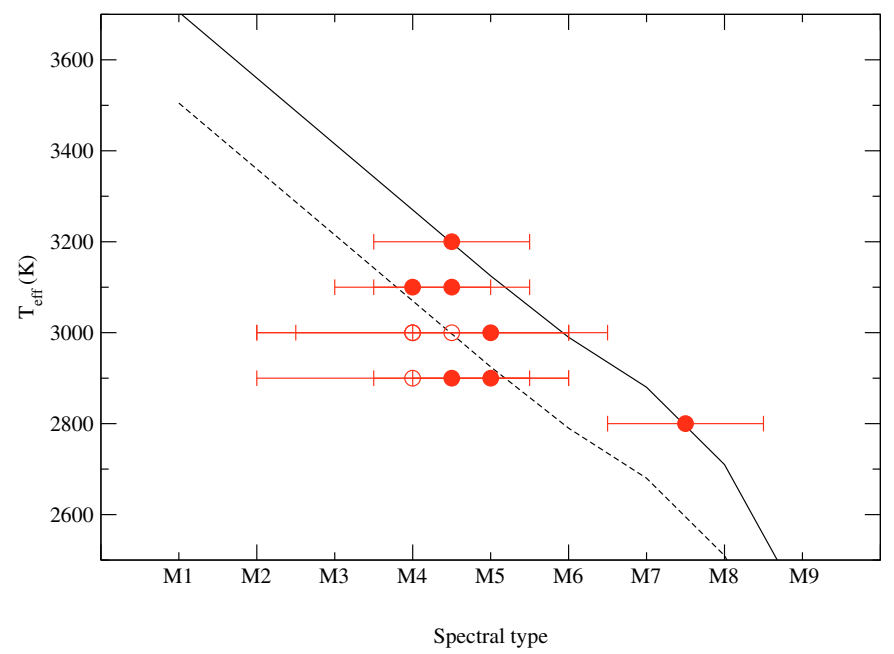

Fig. 2. $T_{\text {eff }}$ vs. spectral type for the previously known members and candidate members of the Corona Australis star-forming region listed in Table 1. Filled and open circles are used for spectral types derived from spectra (Sicilia-Aguilar et al. 2008) and from narrow-band photometry (López Martí et al. 2005), respectively. The solid line is the temperature scale for PMS objects from Luhman (1999) and Luhman et al. (2003). The dashed line is this scale shifted $200 \mathrm{~K}$ towards lower $T_{\text {eff }}$.

residuals below $0.2 \mathrm{mag}$, which is considered acceptable given the accuracy of the photometry. The robustness of the solution is supported by the distribution of $\chi^{2}$ values, which shows a clear minimum at the best-fitting value for each parameter, and a steep rise after the three best fits, especially for $T_{\text {eff }}$. The differences in the residuals between the three best fits are $\leq 0.01$ in most cases.

As discussed by CSL09, poor fits will be obtained for unresolved pairs and for variable stars (because the WFI and 2MASS observations are not simultaneous). In addition, the spectral energy distributions of some objects may be affected by the signatures of accretion and warm circumstellar dust in the visible (veiling and strong emission lines) and near-infrared (infrared excess), leading to an erroneous estimate of the temperature. In both situations, our fits tend to overestimate both the temperature and the extinction (see CSL09 for details).

The mean extinction error was $\Delta A_{V}=0.15$ mag according to the outcome of the fitting procedure, suggesting that most objects in our sample are located in the foreground rather than in the background of the dark cloud. Sicilia-Aguilar et al. (2008) provide independent extinction measurements for some cloud members, which are derived from their spectral types and nearinfrared colors. For the sources in common with that work, our values of $A_{V}$ tend to be higher than those provided by SiciliaAguilar et al. However, the difference is not larger than $0.5 \mathrm{mag}$ for most of the objects and can be attributed to variability and/or to the use of a different extinction law.

The uncertainty in the temperature determination is estimated from the comparison of the three best-fitting results for each object, which generally span a range of $200 \mathrm{~K}$ in effective temperature. We thus take $200 \mathrm{~K}$ as the error in our temperature values. This agrees with the results of Spezzi et al. (2007) and CSL09, who estimated an error of $250 \mathrm{~K}$ on average from the comparison of their fitting results (obtained with the same procedure) with the effective temperatures derived from spectra for a sample of well-known members of Chamaeleon II and Lupus, respectively.

In Fig. 2 we compare our $T_{\text {eff }}$ values with the published spectral types, derived from narrow-band photometry (López Martí et al. 2005) or spectroscopy (Sicilia-Aguilar et al. 2008) for a

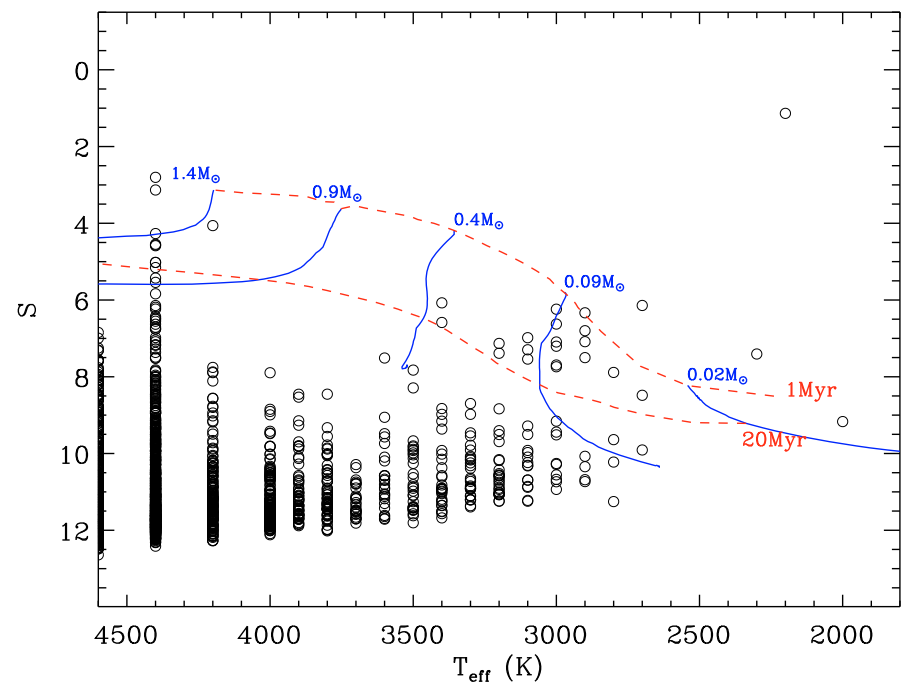

Fig. 3. ( $\left.T_{\text {eff }}, S\right)$ diagram for those sources in our survey with a derived effective temperature below $4500 \mathrm{~K}$. Mass tracks and isochrones from the models of Baraffe et al. (1998) are also plotted.

sample of previously known members and candidate members of Corona Australis, selected in Sect. 3.3 below and listed in Table 1. We also plotted the temperature scale from Luhman (1999) and its extension to late M-type objects by Luhman et al. (2003). As seen in this figure, most of the objects are systematically cooler according to our fitting than the predictions of the Luhman scale. Indeed, other authors have suggested that the true temperatures of PMS stars are lower than the predictions of this temperature scale (e.g. Mohanty \& Basri 2003; Barrado y Navascués et al. 2004), the differences amounting to 150$200 \mathrm{~K}$. Indeed, we find that shifting the Luhman scale $200 \mathrm{~K}$ towards lower temperatures (dashed line in Fig. 2) provides a better agreement with our derived $T_{\text {eff }}$ values. This difference is still within our estimated error range.

\subsection{Contamination}

In Fig. 3 we show the $\left(T_{\text {eff }}, S\right)$ diagram for the sources in our survey. We also plotted two isochrones from the models of Baraffe et al. (1998), corresponding to the expected location of objects of 1 and $20 \mathrm{Myr}$, and several mass tracks from the same models for masses between 1.4 and $0.02 M_{\odot}$. This diagram suggests that no Corona Australis members are present in our survey with temperatures between 4100 and $3400 \mathrm{~K}$, as this range of $T_{\text {eff }}$ is devoid of objects in the area between and above the model isochrones. Indeed, cloud members with $T_{\text {eff }}>3400 \mathrm{~K}$ (spectral types earlier than about M3) are generally expected to be saturated in our optical survey ( $I \lesssim 12$ for a cluster age of $3 \mathrm{Myr}$ and a distance of $130 \mathrm{pc}$, according to the Baraffe et al. (1998) tracks) ${ }^{1}$.

Furthermore, the low density of objects in the young locus together with the clear separation from the rest of sources in our survey suggest that contamination from field stars must be very low in this area of the diagram. Most of the remaining objects are placed well below the $20 \mathrm{Myr}$ isochrone, indicating that they are foreground dwarfs characterized by high values of $S$.

1 As a matter of fact, a couple of known, highly extincted cluster members in this temperature range are actually present in our survey (namely CrA 466 and G-87, both with $A_{V} \gtrsim 8 \mathrm{mag}$ ). However, our fits to their SEDs are not reliable, as they yield too high effective temperatures (around $4400 \mathrm{~K}$ ), probably due to binarity or variability. 
A\&A 515, A31 (2010)

Table 1. SED fitting results for the previously known candidate members of Corona Australis selected with our analysis.

\begin{tabular}{lccccccc}
\hline \hline Name & $A_{V}(\mathrm{mag})$ & $T_{\text {eff }}(\mathrm{K})$ & $S$ & $L\left(L_{\odot}\right)$ & $R\left(R_{\odot}\right)$ & $M\left(M_{\odot}\right)$ & age $(\mathrm{Myr})$ \\
\hline CrA 205 & $0.04 \pm 0.14$ & 3000 & $7.69 \pm 0.07$ & $0.0103 \pm 0.0006$ & $0.376 \pm 0.012$ & 0.072 & 8.0 \\
CrA 452 & $1.14 \pm 0.11$ & 3400 & $6.07 \pm 0.05$ & $0.0755 \pm 0.004$ & $0.79 \pm 0.02$ & 0.4 & 12.7 \\
CrA 453 & $2.00 \pm 0.12$ & 3200 & $7.39 \pm 0.06$ & $0.0177 \pm 0.001$ & $0.433 \pm 0.012$ & 0.11 & 8.0 \\
CrA 465 & $1.42 \pm 0.26$ & 2800 & $7.89 \pm 0.13$ & $0.0065 \pm 0.0008$ & $0.34 \pm 0.02$ & 0.03 & 1.1 \\
CrA 468 & $0.12 \pm 0.15$ & 3100 & $6.98 \pm 0.07$ & $0.0226 \pm 0.0015$ & $0.52 \pm 0.02$ & 0.13 & 8.0 \\
CrA 4107 & $0.73 \pm 0.15$ & 3000 & $6.63 \pm 0.08$ & $0.028 \pm 0.002$ & $0.62 \pm 0.02$ & 0.072 & 2.8 \\
CrA 4108 & $0.16 \pm 0.16$ & 2900 & $6.80 \pm 0.08$ & $0.0205 \pm 0.0015$ & $0.57 \pm 0.02$ & 0.055 & 2.2 \\
CrA 4109 & $0.45 \pm 0.13$ & 3000 & $6.24 \pm 0.06$ & $0.039 \pm 0.002$ & $0.74 \pm 0.02$ & 0.075 & 1.4 \\
CrA 4110 & $0.94 \pm 0.16$ & 3000 & $7.09 \pm 0.08$ & $0.0170 \pm 0.0013$ & $0.50 \pm 0.02$ & 0.07 & 4.0 \\
CrA 4111 & $0.22 \pm 0.22$ & 2900 & $7.50 \pm 0.11$ & $0.0107 \pm 0.0011$ & $0.41 \pm 0.02$ & 0.055 & 5.0 \\
G-14 & $2.18 \pm 0.13$ & 3100 & $7.30 \pm 0.07$ & $0.0169 \pm 0.0010$ & $0.452 \pm 0.014$ & 0.09 & 6.4 \\
G-102 & $1.07 \pm 0.17$ & 2900 & $6.33 \pm 0.08$ & $0.031 \pm 0.002$ & $0.70 \pm 0.03$ & 0.07 & 2.0 \\
CrAPMS 3B & $0.33 \pm 0.15$ & 2700 & $6.14 \pm 0.07$ & $0.028 \pm 0.002$ & $0.77 \pm 0.03$ & 0.04 & 1.1 \\
IRAC-CrA 3 & $1.73 \pm 0.25$ & 2700 & $8.48 \pm 0.13$ & $0.0033 \pm 0.0004$ & $0.261 \pm 0.015$ & 0.03 & 9.0 \\
\hline
\end{tabular}

References. López Martí et al. (2005, and in preparation); Sicilia-Aguilar et al. (2008); Neuhäuser \& Forbrich (2008); Meyer \& Wilking (2009).

Background contamination would be caused by cool giants in the same range of effective temperatures as our objects of interest, but this contamination is expected to be very low due to the high galactic latitude of Corona Australis $\left(b \sim 18^{\circ}\right)$, and to the dark cloud itself.

Following CSL09, the foreground contamination was estimated by computing the amount of main-sequence stars expected in our surveyed area for different temperature bins according to the local initial mass function (IMF) by Chabrier (2003). For $T_{\text {eff }}<3400 \mathrm{~K}$, the number of these objects expected to be found in the young locus in Fig. 3 is three (3). The level of background contamination was estimated with the temperature and radii for cool giants provided by Fluks (1998) and the volume density law from Wainscoat et al. (1992). From this estimation only one (1) background contaminant is expected for $T_{\text {eff }}<3400 \mathrm{~K}$. Because we count 23 objects in the area of interest of the $\left(T_{\text {eff }}, S\right)$ diagram, the amount of expected contamination is about $17 \%$.

\subsection{Object selection}

The procedure to identify candidate cloud members is the same as that outlined in CSL09 and is illustrated in Fig. 4. For four intervals of effective temperature we plotted the expected Sparameter distribution of foreground and background stars. As seen in Fig. 4, the number of foreground stars (dashed lines) decreases with decreasing values of $S$, and with decreasing $T_{\text {eff }}$. On the other hand, the number of background stars (dotted lines) is negligible in all but the highest temperatures.

In general, our histograms are close to the predicted numbers of contaminating stars, although incompleteness due to the limiting magnitudes affects the bins with the highest values of $S$. In those ranges of $S$ where the contamination is expected to be negligible, we interpret an excess of sources as objects that are probable members of the star-forming region. Based on the inspection of Fig. 4 and to minimize the number of contaminants, the following criteria were used to select candidate members of the Corona Australis star-forming region:

$$
\begin{aligned}
& 3100<T_{\text {eff }}<3400 \mathrm{~K}, \quad S<8 \text {; } \\
& 2800<T_{\text {eff }}<3100 \mathrm{~K}, \quad S<9 \text {; } \\
& 2500<T_{\text {eff }}<2800 \mathrm{~K}, \quad S<10 \text {; } \\
& 2200<T_{\text {eff }}<2500 \mathrm{~K}, \quad S<10 .
\end{aligned}
$$

In this way, 4, 13, 5 and 3 candidates respectively were selected in each temperature bin, amounting to 22 in total. The objects were then visually inspected to reject bad detections either due to bad pixels (two objects) or to source confusion in the cross-match (one object). Sources for which the fits derived ages older than expected for Corona Australis members were also rejected as likely contaminants; concretely, we discarded two objects whose derived ages were older than 100 Myr. For another source, the SED fitting suggests an age of $18 \mathrm{Myr}$, which we consider to be slightly too old to be a member of Corona Australis. The residual in the best fit for this latter source is relatively high $\left(\chi^{2} \sim 0.4\right)$ due to its faintness (and hence large photometric errors) in the R-band ( $R>20 \mathrm{mag}$ ). Because we do not have any additional information to support membership for this object, we chose to be conservative and reject it as a cluster member as well.

This left us with 19 objects in the list of selected candidate members of Corona Australis. Thirteen (13) of them had been presented in previous works as candidate members of the region, and eight of these candidates had been confirmed as members through optical or near-infrared spectroscopy (see references in Table 1). Another object (IRAC-CrA 3) had been selected by us as a candidate class II member of Corona Australis in a parallel study based on Spitzer photometry (López Martí et al., in preparation). Our fitting results for these fourteen objects are listed in Table 1: They have effective temperatures between 2700 and $3400 \mathrm{~K}$ and masses betweeen 0.03 and $0.4 M_{\odot}$. Only two objects have masses above $0.1 M_{\odot}$. The optical extinction varies from 0 to $2.2 \mathrm{mag}$.

Together with the previously known candidates, our list still contains five objects that are likely members of the Corona Australis region, all of them with ages younger than $15 \mathrm{Myr}$ according to the SED fitting, and none with reported detections in previous works. They have effective temperatures and masses within the same range as the previously known objects, but tend to be somewhat more extincted, with $A_{V}$ values in the range 14 mag and one object with $A_{V}>6$ mag. The optical and nearinfrared photometry for these new candidate members is given in Table 2, and their parameters from the SED fitting are listed in Table 3.

Note that (excluding the two objects rejected due to photometry problems and the one cross-matching misidentification) we classified three sources as likely contaminants, which agrees very well with our estimation from Sect. 3.2. On the other hand it must be remarked that we are missing members of the region 
B. López Martí et al.: The low-mass diskless population of Corona Australis

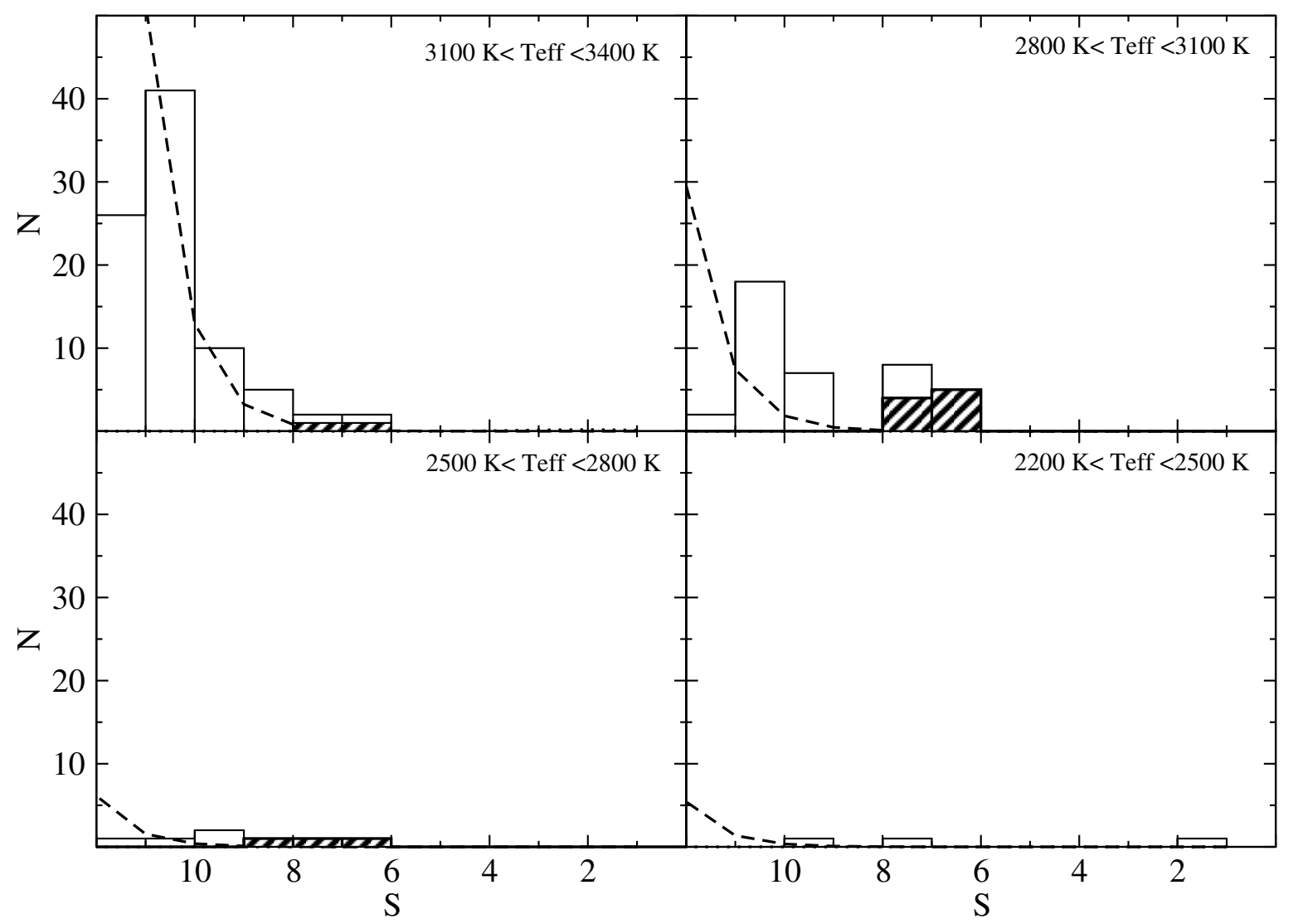

Fig. 4. Histograms of $S$ values at different temperature ranges in the Corona Australis star-forming region. The dashed lines indicate the expected contamination by field main sequence stars, and the hardly visible dotted lines at the bottom of each panel, the contamination by background cool giants. The presence of members of the star forming region is evident in those ranges of $S$ where contamination is expected to be negligible. The hashed histograms show the contribution of objects from previous studies to these excesses.

Table 2. Optical and near-infrared photometry of our new candidate members.

\begin{tabular}{lccccc}
\hline \hline Name & $R$ & $I$ & $J$ & $H$ & $K s$ \\
\hline CrA J190111.6-364532.0 & $18.067 \pm 0.004$ & $15.779 \pm 0.005$ & $13.375 \pm 0.026$ & $12.496 \pm 0.028$ & $12.129 \pm 0.026$ \\
CrA J190139.1-370016.8 & $21.07 \pm 0.06$ & $18.023 \pm 0.006$ & $14.257 \pm 0.029$ & $13.045 \pm 0.033$ & $12.296 \pm 0.026$ \\
CrA J190151.7-371048.4 & $16.451 \pm 0.002$ & $14.500 \pm 0.003$ & $12.326 \pm 0.030$ & $11.423 \pm 0.033$ & $11.047 \pm 0.030$ \\
CrA J190207.6-371156.5 & $19.615 \pm 0.013$ & $17.022 \pm 0.003$ & $14.066 \pm 0.029$ & $13.093 \pm 0.029$ & $12.541 \pm 0.023$ \\
CrA J190239.2-365311.1 & $17.928 \pm 0.005$ & $15.654 \pm 0.005$ & $13.190 \pm 0.024$ & $12.471 \pm 0.022$ & $12.060 \pm 0.023$ \\
\hline
\end{tabular}

Table 3. SED fitting results for our new candidate members of Corona Australis.

\begin{tabular}{lccccccc}
\hline \hline Name & $A_{V}(\mathrm{mag})$ & $T_{\text {eff }}(\mathrm{K})$ & $S$ & $L\left(L_{\odot}\right)$ & $R\left(R_{\odot}\right)$ & $M\left(M_{\odot}\right)$ & age $(\mathrm{Myr})$ \\
\hline CrA J190111.6-364532.0 & $1.78 \pm 0.13$ & 3000 & $7.20 \pm 0.06$ & $0.0161 \pm 0.0009$ & $0.471 \pm 0.013$ & 0.07 & 4.5 \\
CrA J190139.1-370016.8 & $6.20 \pm 0.14$ & 3200 & $7.13 \pm 0.07$ & $0.0224 \pm 0.0014$ & $0.487 \pm 0.016$ & 0.13 & 8.0 \\
CrA J190151.7-371048.4 & $1.96 \pm 0.09$ & 3400 & $6.58 \pm 0.05$ & $0.047 \pm 0.002$ & $0.627 \pm 0.013$ & 0.3 & 14.3 \\
CrA J190207.6-371156.5 & $3.62 \pm 0.13$ & 3100 & $7.54 \pm 0.06$ & $0.0135 \pm 0.0007$ & $0.404 \pm 0.011$ & 0.1 & 10.0 \\
CrA J190239.2-365311.1 & $1.3 \pm 0.2$ & 2900 & $7.08 \pm 0.09$ & $0.0157 \pm 0.0013$ & $0.50 \pm 0.02$ & 0.05 & 3.2 \\
\hline
\end{tabular}

whose $S$-parameter values are in the ranges affected by contamination in the histograms of Fig. 4. Indeed, some excess above the expected foreground contamination is observed in some bins to the left of our $S$ cutoff value for $T_{\text {eff }}<3100 \mathrm{~K}$ (upper panels of Fig. 4), where we would expect to find more cluster members. In addition, as explained in Sect. 3.1, wrong fits would be obtained for objects affected by variability, binarity, strong accretion or infrared excess, and these sources would be wrongly placed in the $\left(T_{\text {eff }}, S\right)$ diagram of Fig. 3. As a consequence, cloud members with primordial disks may be slightly underrepresented in our selected sample.
In particular, other candidate members from the studies of Fernández \& Comerón (2001; 1 object), López Martí et al. (2005; 3 objects), Forbrich et al. (2007; 2 objects) and SiciliaAguilar et al. (2008; 2 objects) are detected in our survey with photometry in all RIJHKs bands, but are not selected with our method. Hence, they are not listed in Table 1 nor plotted in Fig. 1 (but they are included in Figs. 3 and 4). For the previously known candidate members present in our survey, our success rate in recovering them is thus about $65 \%$, or $72 \%$ considering only objects whose membership had been confirmed by spectroscopy; these percentages agree well with the $30 \%$ of members with poor 


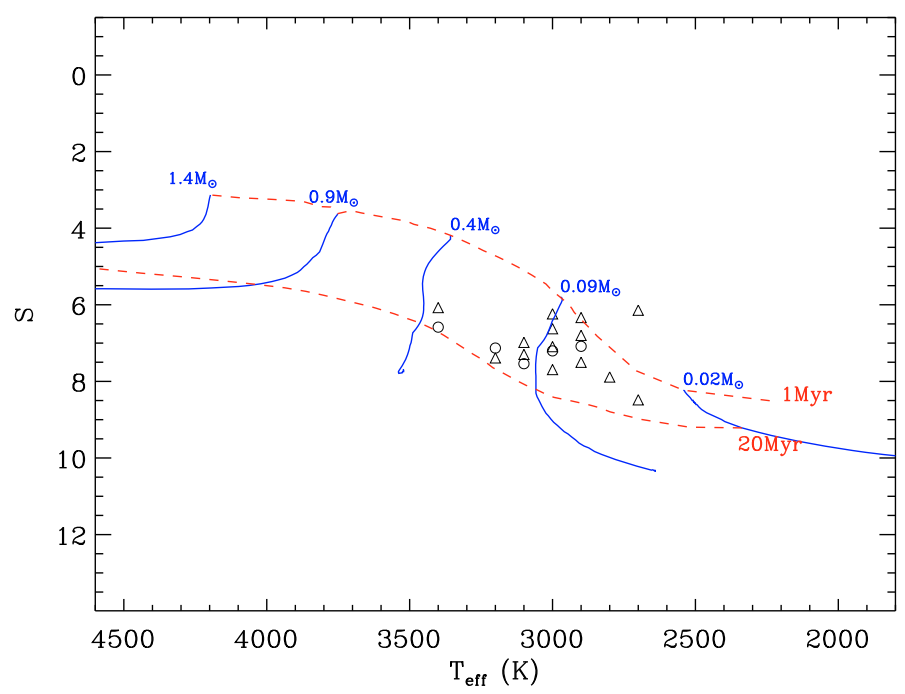

Fig. 5. Same as Fig. 3 for the previous candidate members (triangles) and new candidate members from this work (circles) selected in Sect. 3.3.

fits found in the Lupus clouds (CSL09). For the same reasons there could be some yet unknown members of $\mathrm{CrA}$ which are not selected by our criteria.

\section{Discussion}

\subsection{Membership of the candidates}

We now discuss the membership status of our five objects. Figure 5 is a $\left(T_{\text {eff }}, S\right)$ diagram analogous to Fig. 3, but showing only the location of the members and candidate members of Corona Australis selected in this work.

The spatial distribution of our sources is shown in Fig. 1. Both the previously known candidate members from Table 1 and the new candidate members from Table 3 are located in the northern WFI field; the only exception is the candidate member CrA 205 from López Martí et al. (2005), which is placed in the southern field. This distribution supports the membership of our new candidates to the Coronet cluster around the intermediatemass star R CrA.

Two of our candidates, CrA J190111.6-364532.0 and CrA J190151.7-371048.4, have a USNO-B counterpart within $2^{\prime \prime}$. We compare their proper motions as reported in this catalog with those of previously known members of the Coronet cluster (see Table 4). We considered only USNO-B measurements with a high probability estimator $(\geq 0.7)$ and measurements in at least three epochs (Monet et al. 2003). The proper motion data are summarized in Table 4.

With only 14 objects in total it is not possible to perform a statistically meaningful membership analysis based on proper motion. The upper panel of Fig. 6 shows $\mu_{\delta}$ versus $\mu_{\alpha} \cos \delta$ for all sources. In the lower panel we have plotted the current spatial locations of the sources and their expected displacement in $10^{5} \mathrm{yr}$. While we cannot draw any firm conclusion on their membership, this analysis shows that at least one of the candidates, CrA J190111.6-364532.0, has a proper motion consistent with other members. For CrA J190151.7-371048.4 there is at least some marginal agreement (within the errors) in its proper motion components with respect to those of the majority of cluster members plotted in Fig. 6. Inspection of the optical images shows that this object has two close visual companions, located
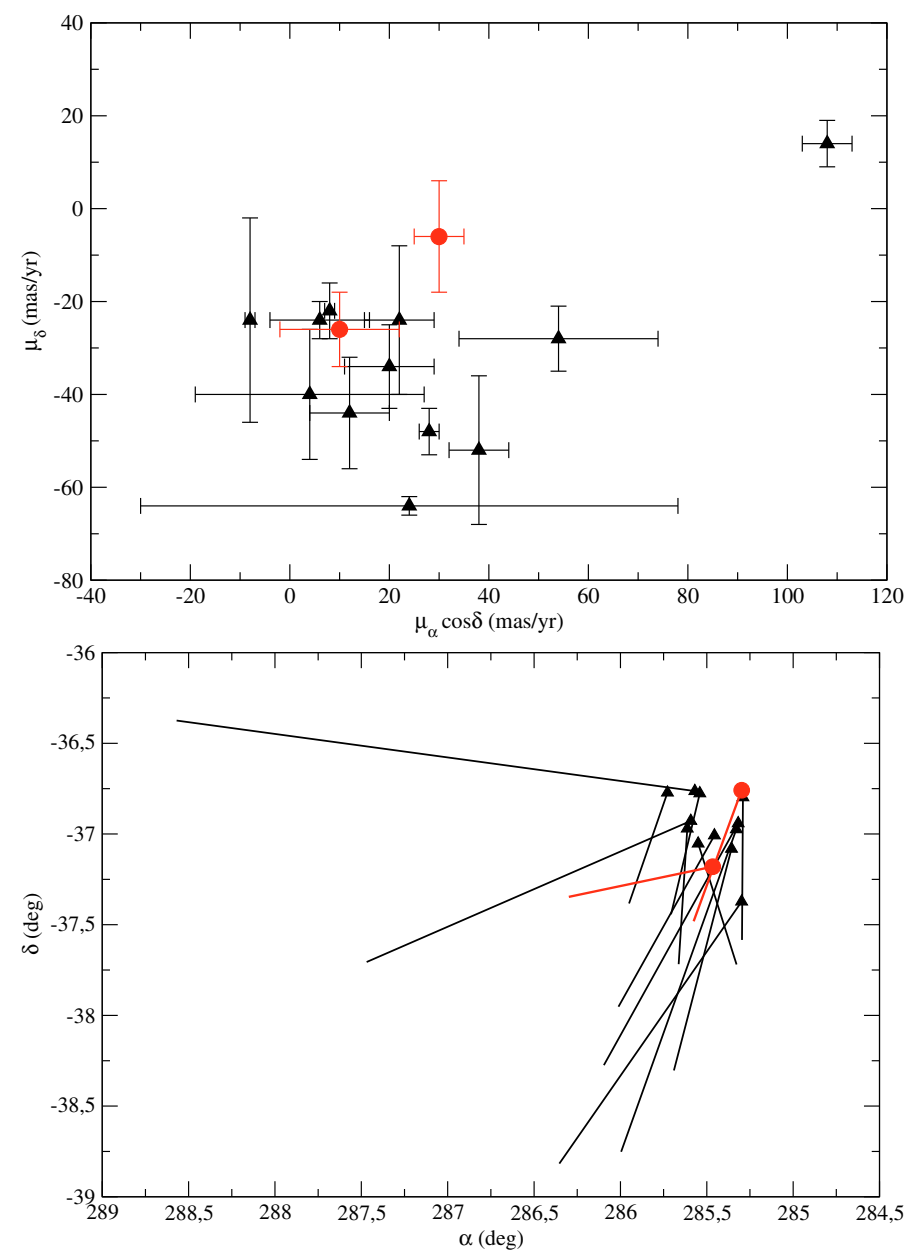

Fig. 6. Upper panel: $\mu_{\delta}$ versus $\mu_{\alpha} \cos \delta$ from the USNO-B1 catalogue for Coronet candidate members from previous works (triangles) and our new candidate members (circles). Lower panel: Spatial location of the same sources. The lines indicate their expected displacement in $10^{5} \mathrm{yr}$.

about $3^{\prime \prime}$ to the NE and $2^{\prime \prime}$ to the NW respectively, a fact that may be affecting the proper motion measurements.

It is remarkable that one source, namely CrA 4109, displays clearly larger proper motion than the rest of probable cluster members plotted in Fig. 6, with $\mu \simeq 109$ mas/yr. The direction of its expected displacement is also significantly different. Given the low object density in our surveyed region, source confusion seems unlikely. Moreover, the USNO-B $R$ and $I$ photometry (16.07 and $13.98 \mathrm{mag}$, respectively) is fully consistent with the WFI photometry for this object $(R=15.99$ and $I=13.98$; López Martí et al. 2005). Hence we are quite confident that our identification of CrA 4109 with this proper motion source is correct. On the other hand, although there is no definitive confirmation of its membership to Corona Australis, there are several indications in the literature ( $\mathrm{H} \alpha$ emission, mid-infrared photometry and spectroscopy) suggestive of CrA 4109 being a young object (López Martí et al. 2005; Sicilia-Aguilar et al. 2008).

In Fig. $1 \mathrm{CrA} 4109$ is located on the outskirts of the cloud core in an area of relatively low extinction. A possibility would thus be that this object was ejected from its birth site due to dynamical interactions with other cluster members, maybe within a multiple system. We note that two more sources (CrA 4107 and CrA 4108) are located in the same area as CrA 4109, but their proper motions agree very well with those of the majority of cluster members. 
B. López Martí et al.: The low-mass diskless population of Corona Australis

Table 4. Proper motion information for CrA members and candidate members.

\begin{tabular}{lccccc}
\hline \hline Name & USNO-B1 & $\mu_{\alpha} \cos \delta(\mathrm{mas} / \mathrm{yr})$ & $\mu_{\delta}(\mathrm{mas} / \mathrm{yr})$ & $\mathrm{P}$ & Nobs \\
\hline CrA 205 & $0526-0958780$ & $38 \pm 6$ & $-52 \pm 16$ & 9 & 4 \\
CrA 466 & $0530-0881950$ & $24 \pm 54$ & $-64 \pm 2$ & 7 & 3 \\
CrA 468 & $0529-0905310$ & $20 \pm 9$ & $-34 \pm 9$ & 9 & 4 \\
CrA 4107 & $0532-0801755$ & $8 \pm 1$ & $-22 \pm 6$ & 9 & 4 \\
CrA 4108 & $0532-0800872$ & $6 \pm 10$ & $-24 \pm 4$ & 9 & 4 \\
CrA 4109 & $0532-0800993$ & $108 \pm 5$ & $14 \pm 5$ & 9 & 4 \\
CrA 4110 & $0530-0881945$ & $28 \pm 2$ & $-48 \pm 5$ & 9 & 4 \\
CrAPMS 3AB & $0530-0882190$ & $54 \pm 20$ & $-28 \pm 7$ & 9 & 4 \\
[FP2007] J190149.35-370028.6 & $0529-0905310$ & $20 \pm 9$ & $-34 \pm 9$ & 9 & 4 \\
G-1 & $0530-0882197$ & $22 \pm 7$ & $-24 \pm 16$ & 9 & 4 \\
G-14 & $0529-0905404$ & $-8 \pm 1$ & $-24 \pm 22$ & 7 & 4 \\
G-102 & $0529-0905023$ & $12 \pm 8$ & $-44 \pm 12$ & 9 & 4 \\
RX J1901.1-3648 & $0532-0800238$ & $4 \pm 23$ & $-40 \pm 14$ & 9 & 4 \\
\hline CrA J190151.7-371048.4 & $0528-0928314$ & $30 \pm 5$ & $-6 \pm 12$ & 9 & 4 \\
CrA J190111.6-364532.0 & $0532-0800263$ & $10 \pm 12$ & $-26 \pm 8$ & 9 & 4 \\
\hline
\end{tabular}

References. Neuhäuser et al. (2000); López Martí et al. (2005); Forbrich \& Preibisch (2007); Sicilia-Aguilar et al. (2008); Neuhäuser \& Forbrich (2008); this work.

Table 5. Spitzer photometry of our new candidate members.

\begin{tabular}{|c|c|c|c|c|c|c|c|c|}
\hline Name & [3.6] & [4.5] & [5.8] & [8.0] & [24.0] & $\alpha_{I R A C}$ & Class & $\operatorname{Disk}^{a}$ \\
\hline CrA J190111.6-364532.0 & $11.653 \pm 0.004$ & & $11.375 \pm 0.015$ & & $8.23 \pm 0.075$ & & & \\
\hline CrA J190139.1-370016.8 & $11.653 \pm 0.005$ & $11.48 \pm 0.006$ & $11.415 \pm 0.016$ & $11.392 \pm 0.026$ & n.d. & -2.556 & III & $\mathrm{A} / \mathrm{N}$ \\
\hline CrA J190151.7-371048.4 & $10.553 \pm 0.003$ & $10.44 \pm 0.003$ & $10.265 \pm 0.007$ & $10.182 \pm 0.008$ & n.d. & -2.397 & III & $\mathrm{A} / \mathrm{N}$ \\
\hline CrA J190207.6-371156.5 & $11.893 \pm 0.004$ & $11.86 \pm 0.006$ & $11.795 \pm 0.017$ & $11.902 \pm 0.03$ & n.d. & -2.836 & III & $\mathrm{N}$ \\
\hline CrA J190239.2-365311.1 & $11.673 \pm 0.004$ & $11.54 \pm 0.005$ & $11.455 \pm 0.015$ & $11.492 \pm 0.018$ & n.d. & -2.633 & III & $\mathrm{N}$ \\
\hline
\end{tabular}

Notes. n.d. $=$ not detected; blank $=$ not observed.

(a) $\mathrm{A}=$ thin disk; $\mathrm{N}=$ no disk (see discussion in text).

\subsection{Disks?}

Given that our candidates from Table 3 had not been detected in previous surveys (the vast majority of them looking for signatures of accretion or disks), they are expected to be diskless, or to have at most optically thin disks. To check this, we cross-matched our optical-NIR catalog with the Spitzer IRAC and MIPS $24 \mu \mathrm{m}$ observations from the study reported in López Martí et al. (in preparation; see that work for details on the reduction and analysis of these data).

The mid-infrared photometry for our new candidates is summarized in Table 5. Four of them have [3.6]-[4.5] and [5.8]-[8.0] colors close to zero, as expected for class III sources (e.g. Allen et al. 2004; Hartmann et al. 2005). With the criterion proposed by Lada et al. (2006) to make a classification of the disks based on the value of the SED slope $\alpha_{I R A C}$ in the 3.6-8.0 $\mu \mathrm{m}$ range, we find that two sources, CrA J190239.2365311.1 and CrA J190207.6-371156.5, are classified as diskless $\left(\alpha_{\text {IRAC }}<-2.56\right)$, while CrA J190151.7-371048.4 and CrA J190139.1-370016.8 could be surrounded by anemic (or thin) disks $\left(-2.56<\alpha_{I R A C}<-1.80\right)$. We remark though that the slope values of the last two objects $(-2.556$ and -2.397 , respectively) are very close to the defined limiting value between anemic disks and no disks. Furthermore these limits are not absolute, because they depend slightly on spectral type and (more strongly) on photometric errors (Lada et al. 2006; Hernández et al. 2008). Given the dispersion on the slope of the field (thus, diskless) objects in our survey $(\sigma \sim 0.1)$, we consider CrA J190151.7-371048.4 and CrA J190139.1-370016.8 as diskless. The remaining source (CrA J190111.6-364532.0) could not be classified according to these schemes, because it was not observed at 4.5 and $8.0 \mu \mathrm{m}$.

The SEDs of our candidates are shown in Fig. 7 compared to the best-fitting model photosphere for each object. We note that the error bars are smaller than the symbol size in the plots due to the low flux uncertainties. From visual inspection, the four sources classified as diskless display nearly photospheric SEDs in the whole range in which they are detected (up to $8 \mu \mathrm{m}$ ).

The source CrA J190111.6-364532.0 is the only one with a counterpart at $24 \mu \mathrm{m}$. As seen in Fig. 7, its SED has the characteristic shape of a star that has cleared its inner disk, showing a clear excess over the photosphere only after $10 \mu \mathrm{m}-$ a so-called "transitional disk" (e.g. Calvet \& D'Alessio 2001; Calvet et al. 2005). This source would have a mass of about $0.07 M_{\odot}$ according to the SED fitting, which would make it one of the lowestmass objects reported to possess a transitional disk to date.

\subsection{Disk fraction and transition disks}

Among the previously known objects selected in Sect. 3.3 and listed in Table 1 there are five class II sources (G-14, CrA 465, CrA 4107, CrA 4110 and IRAC-CrA 3), four class III sources (CrA 453, CrA 468, CrA 4108 and CrA 4111) $)^{2}$ and three

${ }^{2}$ CrA 4111 was classified as a transitional object (in the sense in which
we are using the term here) by Sicilia-Aguilar et al. (2008), who report
the detection of a $24 \mu \mathrm{m}$ excess for this source. Our own analysis of the Spitzer data, however, does not confirm this result: We do not find 


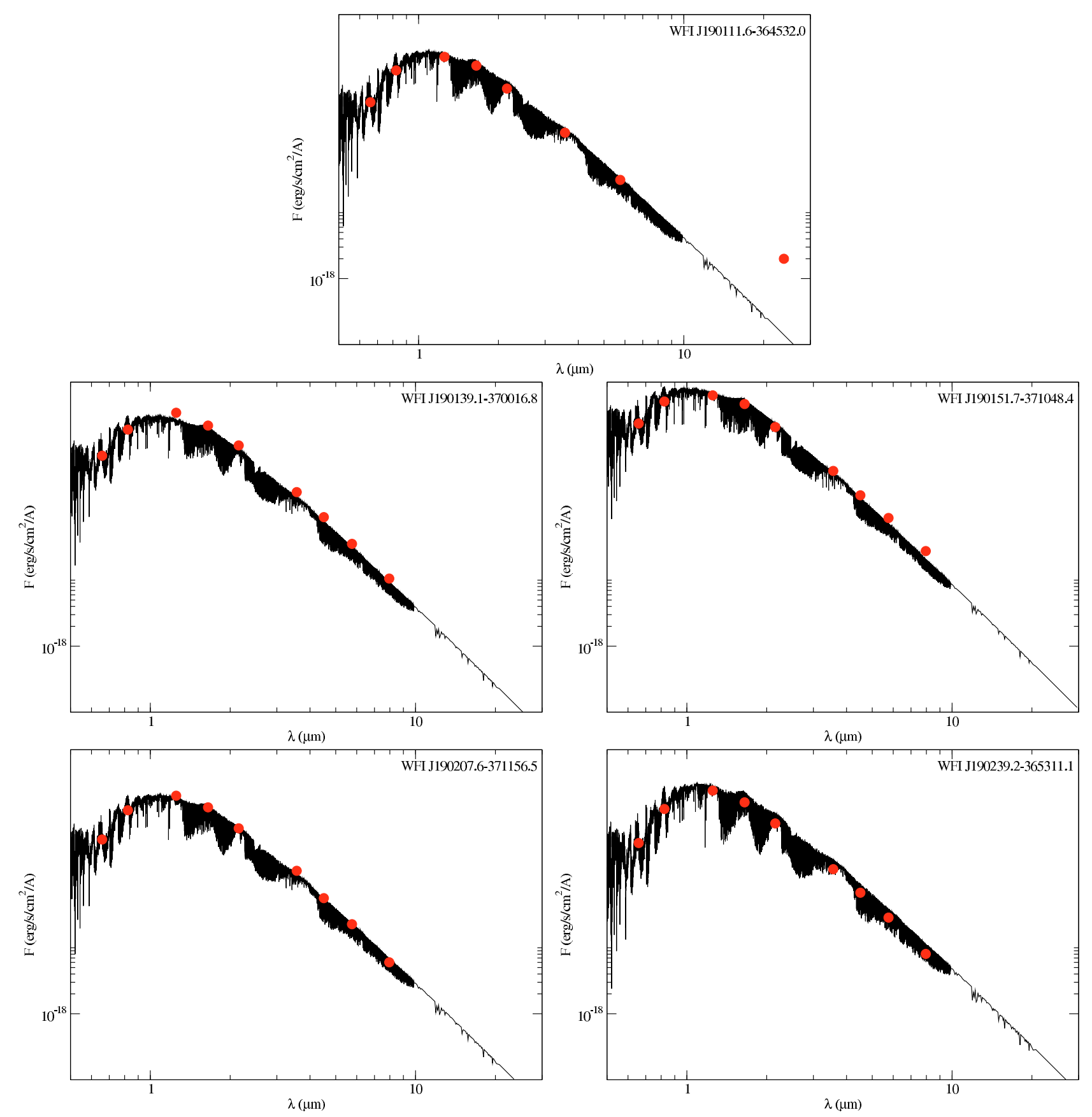

Fig. 7. Spectral energy distributions of our new candidate members of Corona Australis (red dots), from the optical to the mid-infrared, compared to their corresponding best-fitting photospheres according to the SED analysis (see model parameters in Table 3 ).

transitional objects (G-102, CrA 205 and CrA 4109) according to Sicilia-Aguilar et al. (2008) and our own analysis (López Martí et al., in preparation). Note that by "transitional object" we mean here a star or brown dwarf harboring a disk with an inner hole, as suggested by a $24 \mu \mathrm{m}$ excess larger than at $8 \mu \mathrm{m}$ or shorter wavelengths. No classification is available for CrAPMS 3B, because this is the low-mass companion of a PMS star and is unresolved in the IRAC images, nor for $\mathrm{CrA} \mathrm{452,}$ which lies outside the area covered by the IRAC observations.

If we now add our five new candidates to the sample, the final census amounts to five sources with optically thick disks,

any counterpart for CrA 4111 at $24 \mu \mathrm{m}$. Visual inspection of the MIPS $24 \mu \mathrm{m}$ mosaic shows that this object is located in an area of nebulosity, which could have yielded a spurious detection. eight sources without disks, and four sources with transitional disks for a total of 17 classified objects selected following the same criteria. Taking both primordial and transitional disks together, the disk fraction in our sample amounts to $53 \pm 18 \%$, lower than the $70 \%$ reported by Sicilia-Aguilar et al. (2008), due to the increase of diskless objects.

The primordial disk fraction in our sample is $30 \pm 13 \%$, lower than the estimations of Haisch et al. (2001) and Mamajek (2009) for a 3 Myr cluster (the usually quoted age for the Coronet cluster), but not far from their given value of about $50 \pm 10 \%$ when the errors are considered. We note, though, that a relatively large age spread is observed among the objects in our sample, according to the SED fitting results. The derived primordial disk fraction is only slightly higher, within the errors, than that obtained by 
Haisch et al. (2001) for the 5 Myr NGC 2362 cluster (12 $\pm 4 \%$ ), which agrees quite well with the median age of our sample (5 Myr). However, this result should not be interpreted as a new estimation of the cluster age, for several reasons. The first one is of course the low number of objects in our sample and its limited mass range. Second, we caution that individual ages derived from theoretical SED fitting are affected by the many uncertainties still present in the models and may even depend on the accretion history of the objects (e.g. Baraffe et al. 2002, 2009; Mayne \& Naylor 2008). In addition, as explained in Sect. 3, the objects with strongest accretion (thus probably the youngest) are likely to be missed by our selection, which produces a slightly older sample than the mean age of the cluster. The standard deviation of our sample age (3.9) accounts well for these uncertainties.

On the other hand, the ratio of transitional to primordial disks $(45 \pm 22 \%)$ agrees quite well with the $50 \%$ value reported by Sicilia-Aguilar et al. (but see also Ercolano et al. 2009). As already noted by these authors, this ratio is higher than observed in other regions of similar age (around 5-10\%; see e.g. Merín et al., in preparation, for a discussion of the transitional disk ratios in the $c 2 d$ clouds). However, the majority of these studies targeted higher mass stars than considered in Sicilia-Aguilar et al. (2008) and the present study. The larger number of transitional disks among low-mass stars and brown dwarfs with respect to solartype stars could then be interpreted as a hint of a faster disk evolution in the former, as suggested by previous works (e.g. Sterzik et al. 2004; Bouy et al. 2007). It also suggests that the "transitional" stage is not necessarily a short-lived step in the evolution towards a protoplanetary or debris disk, at least for very lowmass objects. However, our sample is too small to derive any statistically meaningful conclusion on this issue.

We remark that the inclusion of the two unclassified objects (CrAPMS 3B and CrA 452) in this census will not alter the measured disk fraction by more than $5 \%$ in either sense (higher or lower disk fraction), nor will it change the ratio of transitional to primordial disks by more than $10 \%$, and hence will not substantially affect these conclusions. On the other hand, as explained in Sect. 3.1, cloud members with primordial disks may be slightly underrepresented in our selected sample. However, even in the most pessimistic case (that would be, if the whole $30 \%$ of badly fitted sources estimated in Sect. 3.1 were surrounded with primordial disks), the disk fractions would still be within the uncertainties of our estimations.

\section{Conclusions}

We performed an analysis of optical and near-infrared data of the Corona Australis star-forming region based on the $S$-parameter formalism. In our surveyed area of $\sim 0.64 \mathrm{deg}^{2}$, we identified fourteen previous candidate members and five new candidate members of this dark cloud.

The new candidates have estimated effective temperatures between 2900 and $3400 \mathrm{~K}$, corresponding to masses between 0.05 and $0.13 M_{\odot}$. They are thus very low-mass stars and massive brown dwarfs. Their ages span between 3 and $15 \mathrm{Myr}$, which is consistent with the reported age spread in Corona Australis $(\sim 1-10 \mathrm{Myr})$, given the uncertainties in the models, in the distance to the cloud and in the SED fitting procedure. The membership of these objects to the star forming region is further supported by their spatial distribution and, when available, proper motion information. The source for which membership is more uncertain is CrA J190151.7-371048.4, the oldest star in our sample according to the SED fitting results.
The SEDs of four of our new candidates are nearly photospheric. The exception is CrA J190111.6-364532.0: This source displays excess at $24 \mu \mathrm{m}$, which is suggestive of a transition disk with an inner hole. With an estimated mass of $0.07 M_{\odot}$, this is one of the lowest-mass objects reported to possess such a disk.

We calculated the disk fraction of the Corona Australis population selected with our method, which is $50 \%$. This value is lower than the one reported in a previous study by SiciliaAguilar et al. (2008). The ratio of transitional to primordial disks (45\%) though agrees well with the fraction of $50 \%$ reported by Sicilia-Aguilar et al. (2008) and is remarkably higher than the value measured in other clusters of similar age. This suggests that transitional disks around brown dwarfs may have longer lifetimes than around low-mass stars. However, this impression should be confirmed with a larger, more statistically meaningful sample.

The results from this work stress the need to properly characterize the diskless population of a region to derive meaningful disk fractions. This is especially important to understand the dependence of the disk fractions with the mass of the central objects, in particular for the very low-mass population. Even in a relatively small survey like the one reported here and in a wellstudied and relatively low-density region like Corona Australis, $25 \%$ of the total number of cloud members selected with our method had not been detected in previous studies based on accretion, disk or activity signatures. An analogous study of an eventual larger-scale survey covering most of the dark cloud would probably identify a significant number of young very low-mass objects belonging to this star-forming region.

Acknowledgements. We are very grateful to F. Comerón for his help with the data analysis and his comments on an early draft of this paper. We also thank A. Bayo for useful discussions.

This work was partially funded by the Spanish MICINN through grants Consolider-CSD2006-00070 and ESP2007-65475-C02-02. It also had funding from the Madrid regional government through grant CAM/PRICIT-S2009ESP1496.

This research is based on observations collected at the European Southern Observatory, on La Silla (Chile), and on data products from the Two Micron All Sky Survey, which is a joint project of the University of Massachusetts and the Infrared Processing and Analysis Center/California Institute of Technology, funded by the National Aeronautics and Space Administration and the National Science Foundation. It also used observations made with the Spitzer Space Telescope, operated by the Jet Propulsion Laboratory, California Institute of Technology, under a contract with NASA.

This publication greatly benefited of the use of the SIMBAD database and VIZIER catalogue service, both operated at CDS (Strasbourg, France). We used the VO-compliant tools Aladin, developed at CDS, TOPCAT, currently developed within the AstroGrid project, and VOSA, developed under the Spanish Virtual Observatory project, and supported by the Spanish MICINN through grant AyA2008-02156.

\section{References}

Allard, F., Hauschildt, P. H., \& Schwenke D. 2000, ApJ, 540, 1005 Allen, L. E., Calvet, N., D'Alessio, P. et al. 2004: ApJS, 154, 363 Baraffe, I., Chabrier, G., Allard, F., \& Hauschildt, P. H. 1998, A\&A, 337, 403 Baraffe, I., Chabrier, G., Allard, F., \& Hauschildt, P. H. 2002, A\&A, 382, 563 Baraffe, I., Chabrier, G., \& Gallardo, J. 2009, ApJ, 702, L27

Barrado y Navascués, D., Mohanty, S., \& Jayawardhana, R. 2004, ApJ, 604, 284 Bouy, H., Brandner,W., Martín, E. L., et al. 2004, A\&A, 424, 213

Bouy, H., Huélamo, N., Martín, E. L., et al. 2007, A\&A, 463, 641 Calvet, N., \& D'Alessio, P. 2001, in Science with the Atacama Large Millimeter Array, ed. A. Wooten, ASP Conf. Proc., 235, 205

Calvet, N., D'Alessio, P., Watson, D. M. et al. 2005, ApJ, 630, L185 Cardelli, J. A., Clayton, G. C., \& Mathis, J. S. 1989, ApJ, 345, 245 Chabrier, G., Baraffe, I., Allard, F., \& Hauschildt, P. H. 2000, ApJ, 542, 464 Chabrier, G. 2003, PASP, 115, 763

Comerón, F., Spezzi, L., \& López Martí, B. 2009, A\&A, 500, 1045

Ercolano, B., Clarke, C. J., \& Robitaille, T. P. 2009, MNRAS, 394, L141

Fernández, M., \& Comerón, F. 2001, A\&A, 380, 264 
A\&A 515, A31 (2010)

Fluks, M. A. 1998, PhD Thesis, Univ. of Amsterdam

Forbrich, J., \& Preibisch, T. 2007, A\&A, 475, 959

Haisch, K. E., Lada, E. A., \& Lada, C. J. 2001, ApJ, 553, L153

Hartmann, L., Megeath, S. T., Allen, L. et al. 2005, ApJ, 629, 881

Hauschildt P. H., Allard F., \& Baron E. 1999, ApJ, 512, 377

Hernández, J., Hartmann, L., Calvet, N. et al. 2008, ApJ, 686, 1195

Knude, J.,, \& Høg, E. 1998, A\&A, 338, 897

Lada, C. J., Muench, A. A., Luhman, K. L. et al. 2006, AJ, 131, 1574

López Martí, B., Eislöffel, J., \& Mundt, R. 2005, A\&A, 444, 175

Luhman, K. L. 1999, ApJ, 525, 466

Luhman, K. L., Stauffer, J. R., Muench, A. A., et al. 2003, ApJ, 593, 1093

Mamajek, E. E. 2009, in Exoplanets and disks: their formation and diversity, AIP Conf. Proc., 1158, 3

Marraco, H. G., \& Rydgren, A. E. 1981, ApJ, 86, 62

Mayne, N. J., \& Naylor, T. 2008, MNRAS, 386, 261

Meyer, M. R., \& Wilking, B. A. 2009, PASP, 121, 350

Merín, B. et al., in preparation

Mohanty, S., \& Basri, G. 2003, ApJ, 583, 451

Monet, D. G., Levine, S. E., Canzian, B. et al. 2003, AJ, 125, 928

Muzerolle, J., Adame, L., D'Alessio, P. et al. 2006, ApJ, 643, 1003
Neuhäuser, R., \& Forbrich, J. 2008, in Handbook of Star Forming Regions, Volume II: The Southern Sky, ed. B. Reipurth, ASP Monograph Publications, 5,735

Neuhäuser, R., Walter, F. M., Covino, E. et al. 2000, A\&AS, 146, 323

Olofsson, G., Huldtgren, M., Kaas, A. A., et al. 1999, A\&A, 350, 883

Prato, L., Greene, T. P., \& Simon, M. 2003, ApJ, 584, 853

Reipurth, B., 2008: Handbook of Star Forming Regions, Volume II: The Southern Sky ASP Monograph Publications, 5

Sicilia-Aguilar, A., Henning, T., Juhász, A. et al. 2008, ApJ, 687, 1145

Skrutskie, M. F., Cutri, R. M., Stiening R. et al. 2006, AJ, 131, 1163S

Spezzi, L., Alcalá, J. M., Frasca, A. et al. 2007, A\&A, 470, 281

Sterzik, M., Pascucci, I., Apai, D. et al. 2004, A\&A, 427, 245

Taylor, K. N. R., \& Storey, J. W. V. 1984, MNRAS, 209, 5

Wainscoat, R. J., Cohen, M., Volk, K. et al. 1992, ApJS, 83, 111

Walter, F. M., Vrba, F. J., Wolk, S. J., et al. 1997, AJ, 114, 1544

Whitworth, A., Bate, M. R., Nordlund, A et al. 2007, in Protostars and Planets V, ed. B. Reipurth, D. Jewitt, \& K. Keil, 459

Wilking, B. A., Greene, T. P., Lada, C. J., et al. 1992, ApJ, 397, 520

Wilking, B. A., McCaughrean, M. J., Burton, M. G. et al. 1997, AJ, 114, 2029 